\title{
Influência do Teor de Umidade na Propriedade de Tenacidade de Espécies Florestais
}

\author{
Denise Ortigosa Stolf ${ }^{1}$, Marília da Silva Bertolini ${ }^{2}$, Fabiane Salles Ferro ${ }^{2}$, \\ André Luis Christoforo ${ }^{3}$, Francisco Antonio Rocco Lahr ${ }^{2}$ \\ ${ }^{1}$ Colégio Trilingüe Inovação, Chapecó/SC, Brasil \\ ${ }^{2}$ Laboratório de Madeiras e de Estruturas de Madeira, Departamento de Engenharia de Estruturas, \\ Escola de Engenharia de São Carlos - EESC, Universidade de São Paulo - USP, São Carlos/SP, Brasil \\ ${ }^{3}$ Departamento de Engenharia Civil - DECiv, Universidade Federal de São Carlos - UFSCAR, São Carlos/SP, Brasil
}

\begin{abstract}
RESUMO
A tenacidade da madeira é uma propriedade mecânica de interesse em situações em que o carregamento por impacto deve ser considerado, encontrando aplicação em silos, pontes e fôrmas para estruturas de concreto armado. Apesar das recomendações do Anexo B - NBR 7190:1997, não tem sido usual a determinação da tenacidade na caracterização da madeira. Este trabalho objetivou investigar a influência do teor de umidade $(30 ; 20 ; 12 ; 6 ; 0 \%)$ na obtenção da tenacidade da madeira angelim-saia (Vatairea sp), Eucalyptus grandis, Pinus elliottii e Corymbia citriodora, considerando a orientação dos anéis de crescimento em relação à direção da força de impacto. Análises estatísticas dos resultados por análise de variância (ANOVA) e teste de Tukey revelaram ser significativa a influência do teor de umidade na tenacidade apenas para a madeira de angelim-saia. Para as demais espécies, a análise estatística demonstrou resultados estatisticamente equivalentes, ou seja, o teor de umidade não foi influente na propriedade de tenacidade. Neste sentido, foi observado que o teor de umidade pode ser significativo na obtenção da tenacidade conforme a espécie e que maiores teores podem fornecer valores superiores desta propriedade.
\end{abstract}

Palavras-chave: madeira, impacto, teor de água.

\section{Influence of the Moisture Content of Some Wood Species on Toughness}

\begin{abstract}
Wood toughness is a mechanical property of interest in structural design where load impact must be considered, as in the construction of silos, bridges and formwork for reinforced concrete structures. However, toughness is not an integral part of the mechanical properties commonly investigated in the characterization of this material. This study aimed to investigate, with the aid of analysis of variance and Charpy pendulum test, the influence of moisture content (30; $20 ; 12 ; 6 ; 0 \%$ ) to obtain the toughness of angelim saia (Vatairea sp), Eucalyptus grandis, Pinus elliottii and Corimbia citriodora wood species, considering the orientation of growth rings in relation to the direction of impact load. Statistical analysis carried out using ANOVA and Tukey test revealed significant influence of moisture content on toughness only with respect to angelim saia wood. Statistical analysis also showed equivalent results for the other three species, that is, moisture content did not significantly influence toughness. Thus, we conclude that moisture content may be significant in obtaining toughness according to the wood species analyzed and higher moisture content can provide higher values of toughness.
\end{abstract}

Keywords: wood, toughness, water content. 


\section{INTRODUÇÃO}

A madeira tem sido muito utilizada pelo homem ao longo da história, estando diretamente relacionada à solução de problemas, como a habitação; a travessia de obstáculos naturais e/ou artificiais; a construção de veículos para os diferentes meios de transporte; o armazenamento e o transporte de produtos agrícolas; a confecção de móveis e utensílios, e a confecção de artefatos esportivos, entre outros (Rocco Lahr et al., 2003).

O conhecimento das propriedades físicas e mecânicas da madeira possibilita seu melhor aproveitamento (Fiorelli \& Dias, 2011; Carreira et al., 2012; Molina et al., 2012; Christoforo et al., 2013). Por causa de dificuldades em realizar ensaios para a caracterização das espécies, muitas vezes a madeira é utilizada sem o conhecimento básico de suas propriedades, levando, assim, ao desperdício desse material (Stolf, 2000).

Especial importância recai sobre o estudo da madeira no que diz respeito ao seu desempenho quando solicitada a ações que provocam impactos (Souza et al., 2009). A obtenção da propriedade de tenacidade da madeira é de extrema relevância, visto que, dependendo da sua utilização, esta deverá ser capaz de suportar condições adversas, como uma sobrecarga ou eventual impacto.

A tenacidade é definida como o trabalho necessário para romper certa amostra à flexão sob uma carga de impacto (Bodig \& Jayne, 1992). A capacidade de um corpo de resistir ao choque depende de sua capacidade de absorver a energia recebida e dissipá-la na forma de deformação (Askeland, 1994). Alguns fatores podem influenciar a resistência ao impacto, tais como a forma e as dimensões dos corpos de prova; os ângulos das fibras; a massa específica; o teor de umidade; a temperatura, e as propriedades anatômicas da madeira (Beltrame et al., 2012).

Devido à natureza anisotrópica e higroscópica da madeira, a caracterização de seu comportamento mecânico exige o conhecimento de suas propriedades dependentes de umidade, uma vez que a madeira, em muitas aplicações, é exposta a diferentes condições climáticas (Ozyhar et al., 2012). Estudos de Gerhards (1968) mostraram que a madeira na condição verde é mais flexível, apresentando maior tenacidade do que a madeira seca. Deste modo, a verificação do aumento da tenacidade proporcionalmente ao teor de umidade pode ser de extrema importância, visto que a madeira, em algumas situações, é submetida a cargas (ações) instantâneas e necessita de maior capacidade de absorção de energia.

Pazos et al. (2003) investigaram a tenacidade e a resistência ao impacto de madeiras de Lysiloma bahamensis, com densidade $0,62 \mathrm{~g} / \mathrm{cm}^{3}$, nas condições seca ao ar (12\%) e saturada. Os valores de tenacidade ou trabalho absorvido $(W)$ obtidos das madeiras secas e saturadas foram iguais a $41,75 \mathrm{~J} \mathrm{e}$ $46,75 \mathrm{~J}$, respectivamente. Dentre outras, os autores concluíram que as madeiras saturadas apresentaram os maiores valores de tenacidade e resistência ao impacto.

Stangerlin et al. (2008a) desenvolveram pesquisa em madeiras de Eucalyptus dunnii com o intuito de investigar a influência do posicionamento das fibras (tangencial e radial) na obtenção da tenacidade e da resistência ao impacto. Os autores concluíram que o posicionamento no sentido radial levou a maiores valores de tais propriedades, quando comparados aos obtidos dos corpos de prova posicionados no sentido tangencial.

Stangerlin et al. (2008b) estudaram a resistência ao impacto em madeiras de Eucalyptus botrioides e Eucalyptus saligna, concluindo não ser possível estimar com segurança a tenacidade da madeira por intermédio da massa específica aparente.

Beltrame et al. (2010) avaliaram, com o uso do pêndulo de Charpy, a resistência ao impacto, o coeficiente de resiliência e a cota dinâmica da madeira de açoita-cavalo (Luehea divaricata Mart. et Zucc Mart.), ensaiada em condições de equilíbrio a $12 \%$ de umidade $\left(0,628 \mathrm{~g} / \mathrm{cm}^{3}\right)$ e saturada $\left(1,076 \mathrm{~g} / \mathrm{cm}^{3}\right)$. Os corpos de prova foram obtidos a partir de árvores procedentes de duas regiões fisiográficas do Estado do Rio Grande do Sul. Também foi investigada, nesta pesquisa, a influência da posição (medula e casca) e do sentido (tangencial e radial) do corpo de prova nos ensaios para obtenção das referidas propriedades. Os resultados encontrados permitiram concluir que a madeira de açoita-cavalo na condição saturada apresentou maior resistência ao impacto e tenacidade $(37,03 \mathrm{~J})$, absorvendo 
grande parte da força aplicada sobre ela quando comparada à resistência e à tenacidade da madeira a $12 \%$ de umidade $(28,87 \mathrm{~J})$. Com relação à posição e ao sentido da orientação dos corpos de prova na obtenção da tenacidade, os resultados da análise estatística revelaram equivalência, implicando não ser significativo o efeito destes dois fatores sobre a tenacidade da madeira de açoita-cavalo.

Beltrame et al. (2012) pesquisaram a influência do teor de umidade (12\% e saturado) na resistência ao impacto da madeira de nogueira-pecã (Carya illinoinensis), procedentes de duas regiões fisiográficas do Estado do Rio Grande do Sul. Os corpos de prova foram preparados e testados quanto aos sentidos da resistência, radial e tangencial, e às posições de retirada, próxima à medula e próxima à casca. Quanto à energia absorvida ou tenacidade, as peças saturadas apresentaram os maiores valores $(7,774 \mathrm{~kg} \cdot \mathrm{m})$, em comparação à tenacidade das madeiras com $12 \%$ de umidade $(2,976 \mathrm{~kg} \cdot \mathrm{m})$, cerca de $161 \%$ superior, em média.

Com o intuito de analisar o comportamento da madeira quando solicitada por forças de impacto, este trabalho objetivou investigar a influência do teor de umidade $(30 ; 20 ; 12 ; 6 ; 0 \%)$ na obtenção da tenacidade em madeiras de angelim-saia (Vatairea sp), Eucalyptus grandis, Pinus elliottii e Corymbia citriodora.

\section{MATERIAL E MÉTODOS}

Os ensaios de tenacidade foram realizados de acordo com os procedimentos recomendados pelo ANEXO B da ABNT NBR 7190:1997 (ABNT, 1997). As amostras de madeira de angelim-saia (AS), com idade superior a 40 anos, foram provenientes do nordeste do Estado de Mato Grosso e as amostras de Pinus elliottii (PE), Eucalipto grandis (EG) e Corymbia citriodora (CC), de 17, 20 e 25 anos, respectivamente, da cidade de Itirapina-SP.

As amostras para ensaios foram retiradas de regiões aleatórias das árvores, levando-se em consideração apenas a obtenção de corpos de prova isentos de defeitos, como preconiza a norma ABNT NBR 7190:1997 (ABNT, 1997). Os corpos de prova foram retirados de modo que os anéis de crescimento estivessem posicionados paralelamente às duas arestas. Para cada uma das espécies investigadas, foram obtidas seis barras isentas de defeito, com dimensões nominais $2 \times 2 \times 200 \mathrm{~cm}$. Cada barra forneceu seis corpos de prova, com dimensões $2 \times 2 \times 30 \mathrm{~cm}$, sendo que um corpo de prova foi utilizado como referência para estimativa do teor de umidade da barra (Figura 1). A partir deste valor e com o controle de peso das amostras (colocadas em climatizadora), os corpos de prova foram condicionados nas porcentagens nominais de umidade 30,20 e $12 \%$. Teores de umidade ao redor de 6 e de $0 \%$ foram obtidos colocando-se as amostras em estufa, com aumento gradativo da temperatura. Com isso, buscou-se reduzir a incidência de defeitos nas amostras. Ressalta-se que, em alguns casos, foram necessários até 25 dias para atingir 0\% de umidade.

Alcançadas as condições desejadas, os 36 corpos de prova por espécie foram ensaiados para a obtenção da tenacidade. Em sua determinação, a orientação dos anéis de crescimento foi tomada como fixa, de maneira a se evitar a sua influência. Para tanto, os ensaios fora realizados de modo que as fibras mais próximas à medula fossem solicitadas à tração. Os testes foram realizados em equipamento desenvolvido por Siqueira (1986) - Figura 2 - com base na máquina tipo FPL (desenvolvida pelo Forest Products Laboratory - EUA), que utiliza o pêndulo de Charpy modificado. O princípio de funcionamento caracteriza-se pela transmissão de energia cinética fornecida pelo pêndulo, através de uma corrente flexível, com um apoio em sua extremidade. A ruptura do corpo de prova é obtida por meio de um único movimento do pêndulo. Os ensaios e cálculos para a determinação da tenacidade foram realizados conforme a norma ASTM D143-52 (ASTM, 1981).

A verificação da influência do teor de umidade (por espécie florestal) no cálculo da tenacidade foi feita por intermédio da análise de variância (ANOVA), no nível de significância (a) de $5 \%$, assumindo a equivalência entre médias como hipótese nula $\left(\mathrm{H}_{0}\right)$ e a não equivalência como hipótese alternativa $\left(\mathrm{H}_{1}\right)$. Note-se que P-valor superior ao nível de significância implica em aceitar a hipótese nula, rejeitando-a em caso contrário.

Quando foi observado ser significativa a influência do teor de umidade nos valores da 


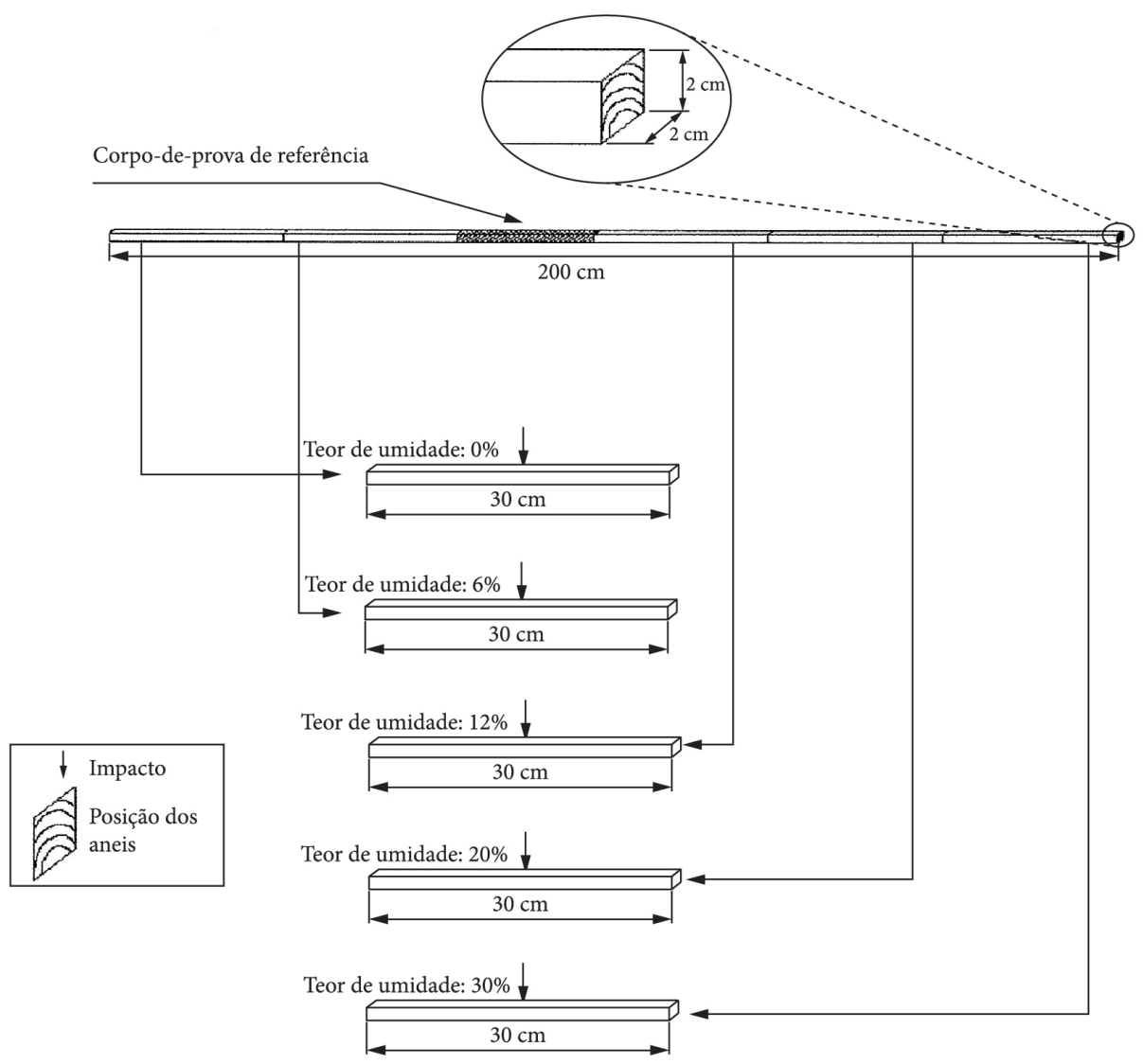

Figura 1. Esquema de obtenção dos corpos de prova para ensaios - teor de umidade e tenacidade da madeira. Fonte: Stolf (2000). Figure 1. Scheme for obtaining samples - moisture content and toughness tests. Source: Stolf (2000).
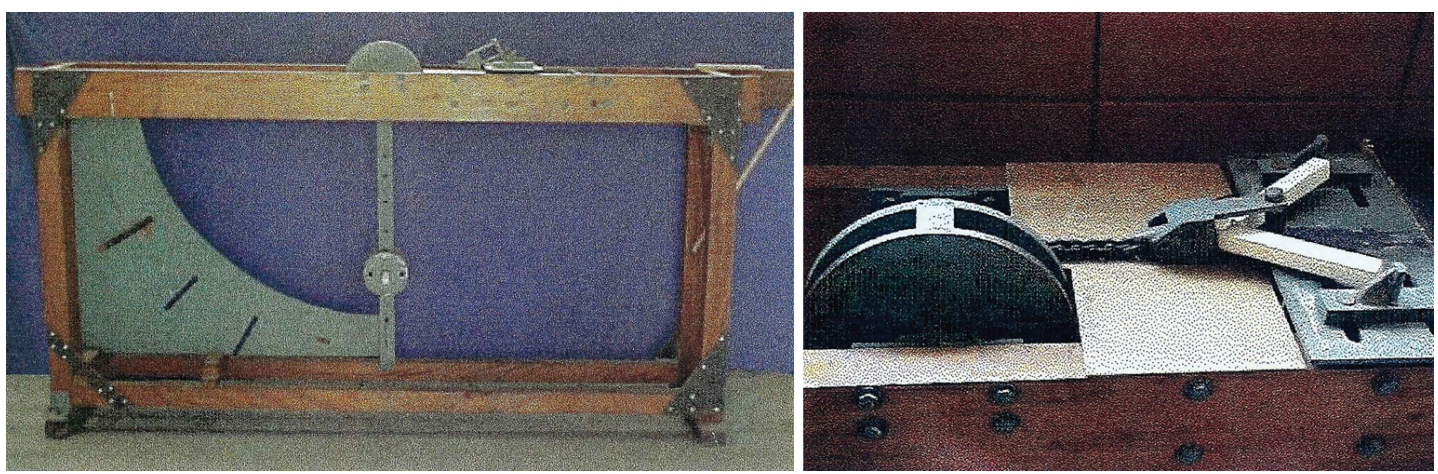

Figura 2. Equipamento para ensaio de tenacidade. Fonte: Stolf (2000).

Figure 2. Toughness testing equipment. Source: Stolf (2000).

tenacidade, foi empregado, na sequência, o teste de comparações múltiplas de Tukey para o agrupamento e a classificação dos grupos.

De forma a validar os resultados obtidos da ANOVA, foram avaliadas a normalidade nas distribuições dos dados (variáveis-resposta) e a homogeneidade entre variâncias, com o auxílio dos testes de Anderson-Darling e Levene, respectivamente.

Para o teste de normalidade de Anderson-Darling, em um nível de significância de 5\%, a hipótese nula consistiu em assumir distribuição normal, para os 
dados, e a não normalidade das distribuições, como hipótese alternativa. Assim, P-valor superior ao nível de significância do teste implica aceitar a hipótese nula (normalidade), refutando-a em caso contrário. Para o teste de Levene, no nível de significância 5\%, a hipótese nula consistiu em assumir equivalência entre variâncias e, para hipótese alternativa, a não equivalência. Observe-se que $\mathrm{P}$-valor superior ao nível de significância implica aceitar a hipótese nula, rejeitando-a em caso contrário.

\section{RESULTADOS E DISCUSSÃO}

A Tabela 1 apresenta os resultados da tenacidade das madeiras em função dos respectivos teores de umidade investigados.

Observou-se, por meio dos valores obtidos para a tenacidade, que, para a espécie Corymbia citriodora, esta propriedade aumenta proporcionalmente com o teor de umidade, no intervalo de 0 a $20 \%$. As demais espécies analisadas possuem tendência semelhante, pela qual ocorre um decréscimo da tenacidade a $12 \%$ de umidade e um aumento em $20 \%$ de umidade. Exceto para as madeiras de angelim-saia e Pinus elliottii, a propriedade de tenacidade novamente tem um decréscimo no teor de $30 \%$ de umidade. Os maiores ganhos para tenacidade foram obtidos para as amostras de angelim-saia e Eucalipto grandis, com 20,43\% e $9,1 \%$, respectivamente, quando considerados o teor de $12 \%$ para $20 \%$ de umidade.

Ghelmeziu (1937/1938) apud Kollmann \& Côté (1968) obteve, para amostras de pinus, um decréscimo da força de impacto, no intervalo de teor de umidade de, aproximadamente, $2,5 \%$ a $12 \%$.
Segundo Kollmann \& Côté (1968), a tenacidade é uma propriedade dependente da força e da flexibilidade. Algumas madeiras tornam-se bastante rígidas, menos flexíveis e frágeis ao se aproximar do estado seco, sendo que o aumento do teor de umidade aumenta a deflexão.

Mesmo sendo utilizadas outras espécies florestais nas pesquisas desenvolvidas por Pazos et al. (2003), Stangerlin et al. (2008a), Beltrame et al. (2010) e Beltrame et al. (2012), os valores de tenacidade foram próximos aos obtidos na presente pesquisa.

Pazos et al. (2003) obtiveram, para a espécie Vatairea lundellii, de mesmo gênero ao qual pertence a amostra de madeira de angelim-saia, objeto deste estudo, valores de tenacidade de, aproximadamente, 19,61 N.m (2 Kg.m) a 12\% de umidade, e de 21,82 N.m (2,225 kg.m) a $30 \%$ de umidade. Observa-se, deste modo, tendência semelhante com valores de tenacidade superiores para maiores teores de umidade.

A Figura 3 apresenta os resultados do teste de normalidade de Anderson-Darling para a tenacidade das quatro espécies investigadas. As espécies Corymbia citriodora (Figura 3b) e de Pinus elliottii (Figura 3d) não apresentaram distribuição normal dos valores de tenacidade, pois a hipótese de normalidade foi rejeitada devido a P-valor $<0,05$. Para possibilitar a realização da análise de variância, os dados devem apresentar uma distribuição normal; logo, se utilizou a transformada de Johnson, a qual ajusta uma distribuição empírica aos dados. As Equações 1 e 2 apresentam as funções de transformação $[\mathrm{Y}(\mathrm{X})]$ obtidas para Corymbia citriodora e de Pinus elliottii, respectivamente.

Tabela 1. Resultados da tenacidade das espécies florestais em função dos teores de umidade.

Table 1. Results tenacity of wood species depending on moisture content.

\begin{tabular}{|c|c|c|c|c|c|c|c|c|c|c|}
\hline \multirow{4}{*}{ Espécie } & \multicolumn{10}{|c|}{ Tenacidade (N.m) } \\
\hline & \multicolumn{10}{|c|}{ Teor de Umidade } \\
\hline & \multicolumn{2}{|c|}{$0 \%$} & \multicolumn{2}{|c|}{$6 \%$} & \multicolumn{2}{|c|}{$12 \%$} & \multicolumn{2}{|c|}{$20 \%$} & \multicolumn{2}{|c|}{$30 \%$} \\
\hline & $\bar{x}$ & $\mathrm{Cv}$ & $\bar{x}$ & $\mathrm{Cv}$ & $\bar{x}$ & $\mathrm{Cv}$ & $\bar{x}$ & $\mathrm{Cv}$ & $\bar{x}$ & $\mathrm{Cv}$ \\
\hline Angelim-saia & 29,33 & 18 & 31,55 & 14 & 30,79 & 12 & 37,08 & 14 & 37,18 & 6 \\
\hline Corymbia citriodora & 54 & 19 & 56,87 & 21 & 59,29 & 11 & 60,54 & 18 & 58,84 & 20 \\
\hline Eucalipto grandis & 37,51 & 20 & 39,87 & 17 & 38,51 & 13 & 42,01 & 9 & 41,17 & 13 \\
\hline Pinus elliottii & 22,04 & 12 & 23,19 & 18 & 22,82 & 8 & 23,75 & 6 & 24,69 & 14 \\
\hline
\end{tabular}

$\bar{x}=$ média amostral; $C v=$ coeficiente de variação. 
(a)

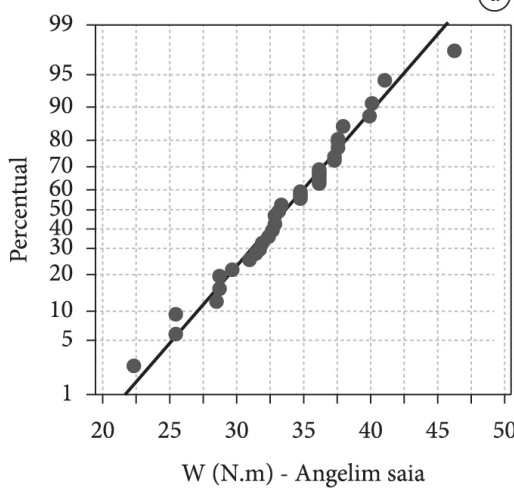

(c)

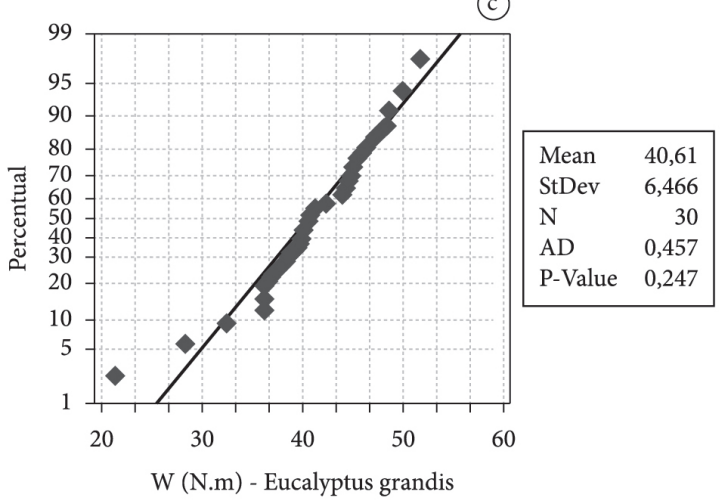

(b)

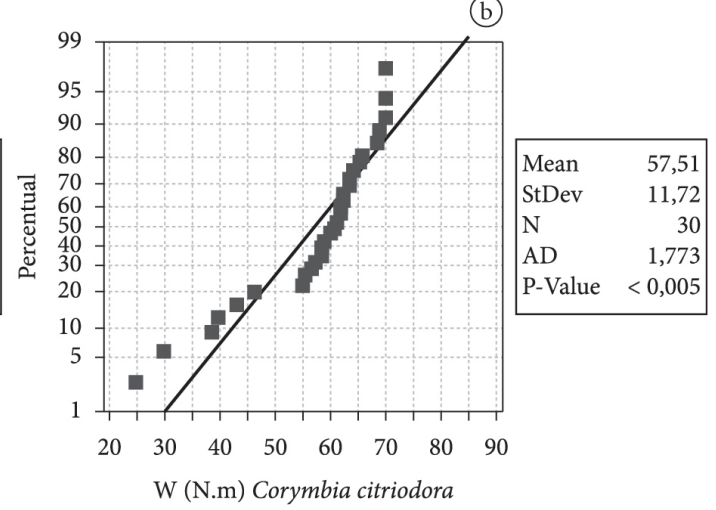

(d)

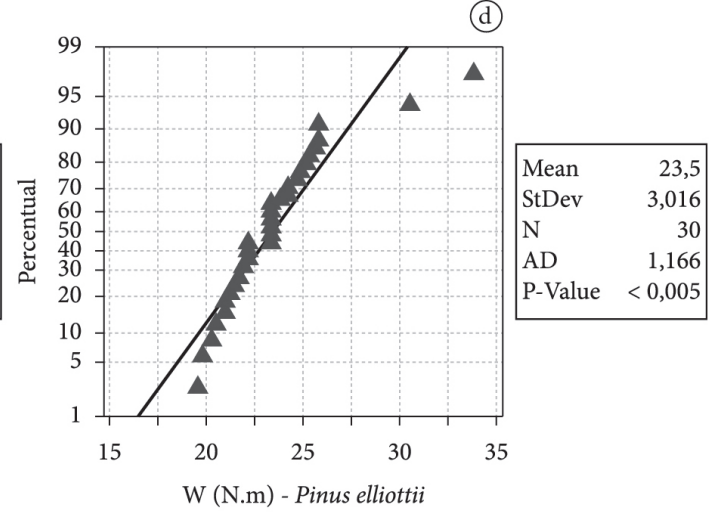

Figura 3. Resultados do teste de normalidade de Anderson-Darling.

Figure 3. Test results of the Anderson-Darling normality.

$Y(X)=0,542051+0,723982 \cdot A \sinh \left(\frac{X-64,1404}{3,63072}\right)$

$Y(X)=-0,649436+1,22757 \cdot A \sinh \left(\frac{X-21,7144}{2,20894}\right)$

A Figura 4 apresenta os resultados do teste de normalidade de Anderson-Darling para os valores de tenacidade das madeiras de Corymbia citriodora (Figura 4a) e de Pinus elliottii (Figura 4b), segundo a transformada de Johnson, evidenciando a eficiência destas transformações realizadas (P-valor $>0,05)$. Com isso, os valores de tenacidade resultaram em uma distribuição normal, possibilitando a análise estatística destes dados pela Análise de Variância e pelo teste de Tukey.

A Tabela 2 apresenta os resultados do teste de Levene para verificação da equivalência entre variâncias, completando, por validar o modelo da ANOVA, e os resultados da análise de variância do fator teor de umidade sobre a tenacidade das madeiras. Os P-valores encontrados, com significância estatística para valores menores do que 0,05 , mostraram que o teor de umidade influiu significativamente na tenacidade apenas para a madeira de angelim-saia, fornecendo resultados equivalentes para as demais espécies de avaliadas.

A Tabela 3 apresenta os resultados do teste de comparações múltiplas de Tukey do fator teor de umidade sobre a tenacidade das amostras de madeiras de angelim-saia. Letras iguais confirmam tratamentos com médias equivalentes.

Da Tabela 3, constata-se que os maiores valores da tenacidade da madeira de angelimsaia foram provenientes dos dois maiores valores do teor de umidade, 20 e 30\%, apresentando valores estatisticamente equivalentes entre si. A propriedade de tenacidade obtida no intervalo de teores de umidade de 0 a $12 \%$ apresentaram diferença estatística em relação aos valores da citada propriedade para teores de umidade de $20 \%$ 

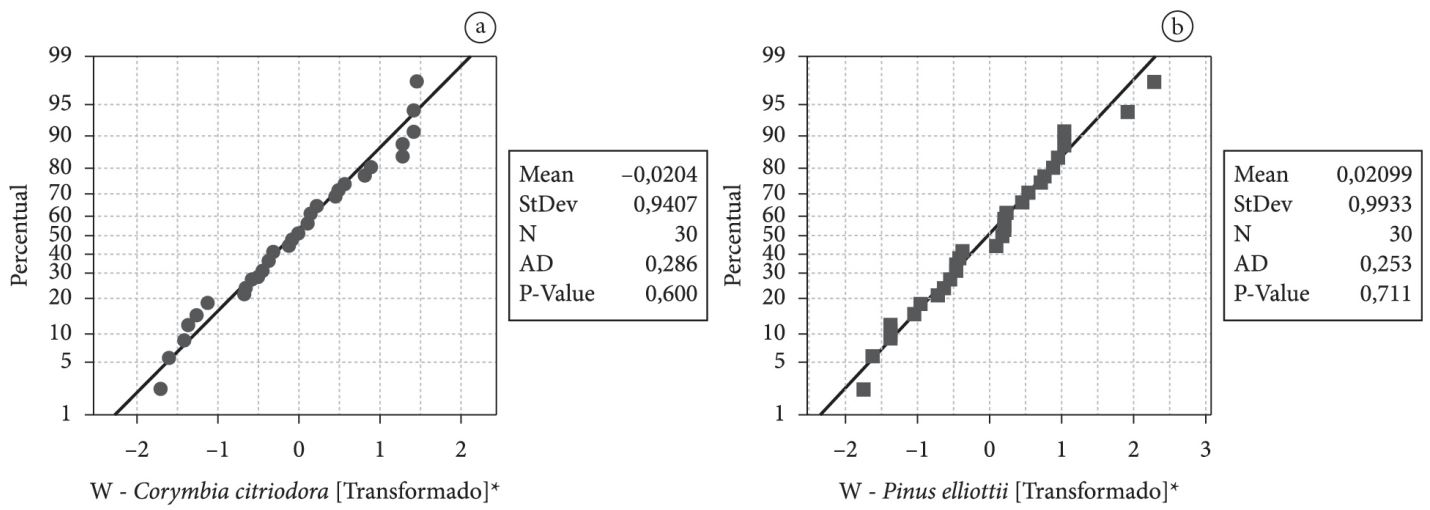

Figura 4. Resultados do teste de normalidade para os dados transformados; ${ }^{*}$ Transformada de Johnson.

Figure 4. Results of normality for the transformed data; ${ }^{\star}$ Johnson Transformation.

Tabela 2. Resultados do teste de Levene e da ANOVA.

Table 2. Results of Levene's test and ANOVA.

\begin{tabular}{lccc}
\multicolumn{1}{c}{ Espécies } & P-valor (Levene) & GL (ANOVA) & P-valor (ANOVA) \\
\hline Angelim-saia & 0,745 & 29 & $\underline{0,007}$ \\
\hline Corymbia citriodora & 0,178 & 29 & 0,477 \\
Eucalipto grandis & 0,820 & 29 & 0,138 \\
Pinus elliottii & 0,528 & 29 & 0,494 \\
\hline
\end{tabular}

Tabela 3. Resultados do teste de Tukey para a madeira de angelim-saia.

Table 3. Tukey test results for Angelim Saia wood.

\begin{tabular}{lccccc} 
& \multicolumn{5}{c}{ Angelim-saia } \\
\cline { 2 - 6 } Tenacidade & \multicolumn{5}{c}{ Teor de Umidade } \\
\cline { 2 - 6 } & $\mathbf{0 \%}$ & $\mathbf{6 \%}$ & $\mathbf{1 2 \%}$ & $\mathbf{2 0} \%$ & $\mathbf{3 0} \%$ \\
\hline $\bar{x}(\mathrm{~N} \cdot \mathrm{m})$ & 29,33 & 31,55 & 30,79 & 37,08 & 37,18 \\
Grupo & $\mathrm{B}$ & $\mathrm{B}$ & $\mathrm{B}$ & $\mathrm{A}$ & $\mathrm{A}$ \\
\hline
\end{tabular}

e 30\%, possibilitando-se observar que o aumento significativo estatisticamente desta propriedade ocorre para os teores de umidade a partir de $20 \%$. Segundo Majano-Majano et al. (2012), para teores de umidade mais elevados, mais energia por unidade de superfície é necessária para separar uma amostra de madeira em duas metades. A energia necessária para iniciar o processo de zona para propagação de trinca aumenta devido à alta ductilidade da madeira com maior teor de umidade, em que mais energia é consumida em fibras de transição por trás da ponta da trinca, além de a dissipação de energia aumentar em razão da deformação irreversível.

Esta tendência também foi observada por Pazos et al. (2003), Beltrame et al. (2010) e Beltrame et al. (2012), os quais constataram que o valor da energia absorvida - ou a tenacidade - para as madeiras saturadas de água apresentou valores superiores quando comparados às tenacidades das madeiras com 12\% de teor de umidade. Assim, observa-se que os resultados obtidos para as amostras de madeira de angelim-saia da presente pesquisa se mostram em conformidade com a literatura, no que diz respeito a maiores valores de tenacidade estarem associados aos maiores valores do teor de umidade.

\section{CONCLUSÕES}

Os resultados obtidos revelaram que os teores de umidade investigados foram influentes ou significativos estatisticamente na tenacidade apenas para a madeira de angelim-saia, cujos valores a $20 \mathrm{e}$ $30 \%$ de umidade foram superiores. Para as demais espécies, os resultados foram equivalentes, isto é, não foi significativa a influência da variação do teor de umidade na tenacidade das madeiras de Eucalipto grandis, Pinus elliottii e Corymbia citriodora.

Em linhas gerais, pode-se concluir que a tenacidade pode ser afetada pelo teor de umidade, dependendo do tipo de espécie investigada, e que o aumento do teor de umidade pode provocar aumentos na tenacidade da madeira. 


\section{STATUS DA SUBMISSÃO}

Recebido: 18 nov., 2013

Aceito: 17 jul., 2014

Publicado: 21 nov., 2014

\section{AUTOR(ES) PARA CORRESPONDÊNCIA}

\section{Marília da Silva Bertolini}

Laboratório de Madeiras e de Estruturas de Madeira, Departamento de Engenharia de Estruturas, Escola de Engenharia de São Carlos - EESC, Universidade de São Paulo - USP , CEP 13560-590, São Carlos, SP, Brasil e-mail: marilia.bertolini@usp.br

\section{REFERÊNCIAS}

American Society for Testing and Materials - ASTM. ASTM D143-52: standard methods of testing small clear specimens of timber. Philadelphia; 1981.

Askeland DR. The science and engineering of materials. 3rd ed. Boston: PWS Publishing Company; 1994.

Associação Brasileira de Normas Técnicas - ABNT. NBR 7190: projeto de estruturas de madeira. Rio de Janeiro; 1997.

Beltrame R, Gatto DA, Modes KS, Stangerlin DM, Trevisan R, Haselein CR. Resistência ao impacto da madeira de açoita-cavalo em diferentes condições de umidade. Cerne 2010; 16(4): 499-504.

Beltrame R, Mattos BD, Gatto DA, Lazarotto M, Haselein CR, Santini EJ. Resistência ao impacto da madeira de nogueira-pecã em diferentes condições de umidade. Ciência Rural 2012; 42(9): 1583-1587. http:// dx.doi.org/10.1590/S0103-84782012005000060

Bodig J, Jayne BA. Mechanical of wood and wood composites. New York: Van Nostrand Reinhold; 1992.

Carreira MR, Segundinho PGA, Rocco Lahr FA, Dias AA, Calil C Jr. Bending stiffness evaluation of Teca and Guajará lumber through tests of transverse and longitudinal vibration. Acta Scientiarum Technology 2012; 34(1): 27-32. http://dx.doi.org/10.4025/ actascitechnol.v34i1.10728

Christoforo AL, Ribeiro SLM Fo, Panzera TH, Rocco Lahr FA. Metodologia para o cálculo dos módulos de elasticidade longitudinal e transversal em vigas de madeira de dimensões estruturais. Ciência Rural 2013; 43(4): 610-615. http://dx.doi.org/10.1590/S010384782013000400008

Fiorelli J, Dias AA. Glulam beams reinforced with FRP externally-bonded: theoretical and experimental evaluation. Materials and Structures 2011; 44(8): 14311440. http://dx.doi.org/10.1617/s11527-011-9708-y
Gerhards CC. Effects of type of testing equipment ans specimen size on toughness of wood. Madison: Forest Service, Forest Products Laboratory; 1968. p. 1-12. Research Paper FPL, 97.

Kollmann FFP, Côté WA. Principles of wood science and technology: solid wood. New York: Springer Verlag; 1968. http://dx.doi.org/10.1007/978-3-642-87928-9

Majano-Majano A, Hughes M, Fernandez-Cabo JL. The fracture toughness and properties of thermally modified beech and ash at different moisture contents. Wood Science and Technology 2012; 46(1-3): 5-21. http://dx.doi.org/10.1007/s00226-010-0389-4

Molina JM, Calil C Jr, Kimura EFA, Pinto EM, Regobello R. Análise numérica do comportamento de elementos de madeira em situação de incêndio. Floresta e Ambiente 2012; 19(2): 162-170. http://dx.doi. org/10.4322/floram.2012.019

Ozyhar T, Hering S, Niemz P. Moisture-dependent elastic and strength anisotropy of European beech wood in tension. Journal of Materials Science 2012; 47(16): 61416150. http://dx.doi.org/10.1007/s10853-012-6534-8

Pazos GB, Morales RPZ, Candelaria VRO. Correlación de los resultados de impacto o tenacidad de 16 maderas mexicanas utilizando dos métodos de prueba. Revista Madera y Bosques 2003; 9(1): 55-70.

Rocco Lahr FA, Calil C Jr, Dias AA. Dimensionamento de elementos estruturais de madeira. Rio de Janeiro: Manole; 2003.

Siqueira ML. Projeto e construção de máquina para determinação da tenacidade de madeira [dissertação]. São Carlos: Escola de Engenharia de São Carlos, Universidade de São Paulo; 1986.

Souza JT, Machado WG, Beltrame R, Modes KS, Vivian MA, Haselein CR. Estudo do comportamento da flexão dinâmica em função da massa específica da madeira de Araucaria angustifolia (Bertol.) Kuntze. In: Anais do XVIII Congresso de Iniciação Científica (CIC), XI Encontro de Pós-Graduação (ENPOS) e a I Mostra Científica; 2009; Pelotas. CD-ROM.

Stangerlin DM, Melo RR, Domingues JMX. Determinação da resistência ao impacto para as madeiras de Eucalyptus dunnii, Corymbia citriodora e Pouteria pachycarpa. In: Anais do XI Encontro Brasileiro em Madeira e Estruturas de Madeira; 2008a; Londrina. CD-ROM.

Stangerlin DM, Melo RR, Santini EJ, Gatto DA, Domingues JMX, Calegari L et al. Avaliação da resistência ao impacto para as madeiras de Eucalyptus botrioides e Eucalyptus saligna. In: Anais do X Congresso Florestal Estadual; 2008b; Nova Prata. CD-ROM.

Stolf DO. Tenacidade da madeira [dissertação]. São Carlos: Escola de Engenharia de São Carlos, Universidade de São Paulo; 2000. 100 p. 